\title{
Evolutionary topology optimization of continuum structures under uncertainty using sensitivity analysis and smooth boundary representation
}

\author{
Jesús Martínez-Frutos*, David Herrero-Pérez \\ Computational Mechanics and Scientific Computing Group, Technical University of Cartagena, \\ Campus Muralla del Mar, 30202 Cartagena, Murcia, Spain
}

\begin{abstract}
This paper presents an evolutionary approach for the Robust Topology Optimization (RTO) of continuum structures under loading and material uncertainties. The method is based on an optimality criterion obtained from the stochastic linear elasticity problem in its weak form. The smooth structural topology is determined implicitly by an iso-value of the optimality criterion field. This isovalue is updated using an iterative approach to reach the solution of the RTO problem. The proposal permits to model the uncertainty using random variables with different probability distributions as well as random fields. The computational burden, due to the high dimension of the random field approximation, is efficiently addressed using anisotropic sparse grid stochastic collocation methods. The numerical results show the ability of the proposal to provide smooth and clearly defined structural boundaries. Such results also show that the method provides structural designs satisfying a trade-off between conflicting objectives in the RTO problem.
\end{abstract}

Keywords: Evolutionary topology optimization, Robust design, Random fields, Optimality criteria.

\section{Introduction}

Topology optimization aims at finding the optimal layout of material within a design domain for a given set of boundary conditions such that the resulting material distribution meets a set of performance targets [1]. Contrary to other disciplines within structural optimization such as size and shape optimization, in topology optimization the material distribution is obtained without assuming any prior structural configuration. This provides a powerful tool to find the best conceptual design that fulfills the requirements at the early stages of the structural design [2]. Such a method has been successfully applied to a wide range of problems, from nanophotonics design [3] to aircraft and aerospace structural design $[4,5]$, which validates it as an effective tool for least-weight and performance design.

Topology optimization methods can be broadly classified, following [6], into density-based methods [7, 8], level set methods $[9,10]$, phase field methods $[11,12]$, topological derivative methods [13, 14] and evolutionary approaches [15]. The variants of Evolutionary Structural Optimization (ESO) method [16] are some of the approaches included in the last category, such as the Bi-directional Evolutionary Structural Optimization (BESO) method [17] and the Evolutionary Topology Optimization (ETO) method using isolines [18, 19, 20] and smoothing boundary representation [21]. These optimization methods are based on heuristic rules including from simple hard-kill strategies (elements with lowest strain energy density are removed)

\footnotetext{
${ }^{*}$ Corresponding author

Email addresses: jesus.martinez@upct.es (Jesús Martínez-Frutos), david.herrero@upct.es (David Herrero-Pérez)
}

to bidirectional schemes (elements can be reintroduced if considered rewarding). Apart from intuition, such methods can use standard adjoint gradient analysis and filtering techniques to stabilize algorithms and results [22, 23].

ETO methods have shown their ability for providing structurally sound and aesthetically pleasing designs [24], which commonly mimic nature's own evolutionary optimization process. Such methods normally assume deterministic conditions to integrate function and form in a synergistic way [25], which obviates the different sources of uncertainty that may affect not only the safety and reliability of structures but also their performance. These sources of uncertainty include epistemic uncertainties, typically due to limited data and knowledge, and aleatory uncertainties, which are the natural randomness in a process, including manufacturing imperfections, unknown loading conditions, variations of the material properties, etc. The introduction of uncertainty to model realistic conditions in the design process has shown to be a key issue for solving realworld engineering problems in several fields, such as civil [26], automotive [27] and mechanical [28] engineering, to name but a few. This fact, together with the development of probabilistic uncertainty propagation methods, has fostered the interest for considering uncertainty within the topology optimization problems, giving rise to the formulation of several approaches embraced under the term of Topology Optimization Under Uncertainty (TOUU) methods.

TOUU methods can be broadly classified, according to the representation and treatment of uncertainties, into nonprobabilistic and probabilistic approaches. Non-probabilistic approaches [29] do not require the statistical information about the uncertainty of the phenomenon but a qualitative notion 
about its magnitude. The worst-case approach [30, 31], taking the form of a min-max optimization problem, and fuzzy techniques [32], making use of fuzzy set theory, are some of the methods included in this category. The main drawback of these approaches is that they are often too conservative, due to overestimation of uncertainty, and may lead to optimal designs with poor structural performance. Conversely, probabilistic methods make use of a probabilistic characterization of the uncertainty of the phenomenon. Several formulations have been proposed in this context, which differs from each other in the design of the objective function as well as in the way the uncertainty is incorporated in the formulation.

Reliability-Based Topology Optimization (RBTO) aims at determining the best design solution with respect to prescribed criteria, e.g. stiffness, weight and construction costs, while explicitly considering the unavoidable effects of uncertainty. This is done by defining the constraints in terms of the probability of constraint violation (probability of failure) [33]. Risk-Averse Topology Optimization (RATO) [34, 35] aims at minimizing a risk cost function that quantifies the expected loss related to the damages, such as excess probability. That is, whereas RBTO provides optimal designs in terms of deterministic prescribed criteria with enough reliability level, RATO provides the best design from the point of view of risk-aversion [36]. Contrary to RBTO and RATO formulations, Robust Topology Optimization (RTO) incorporates statistical moments of the compliance to the objective function. The aim is to obtain optimal designs which are less sensitive to variations in the input data. Several developments based on RTO formulation have been developed to handle uncertainty in loading [37], material [38], stiffness [39], geometry [40], boundary [41], and loading and material [42, 43]. For the specific case of uncertainty in loading, Alvarez and Carrasco [44] and then Dunning et al. [45] showed that the RTO problem of minimizing the expected compliance is analogous to a multiload-like problem associated with a particular finite set of loading scenarios, which depend on the mean and the variance of the perturbations [46]. Nevertheless, a very common practice is to use the weighted sum of the first two statistical moments of compliance as the objective function of RTO formulation [43, 38, 37]. This is the formulation adopted in this work, where the expected value and the standard deviation of the compliance are considered as a measure of structural robustness.

Despite the fact that ETO methods have been successfully applied to the design of many complex industrial deterministic problems, they have not been used to the same extent to address TOUU problems. Kim et al. [47] addressed the RBTO problem using the ESO method and first-order reliability approach, as approximate probability integration method, to solve problems with uncertainty in loading and material. Eom et al. [48] made use of an improved hard-kill BESO method using a response surface to compute the reliability index for addressing RBTO problems with uncertainty in loading and material. The BESO method using a performance measure, with probabilistic constraints formulated in terms of the reliability index, was used by Cho et al. [49] to address RBTO multi-objective problems including uncertainty in static stiffness of bending, torsion, and natural frequency. The linear elasticity hypothesis was exploited by Kanakasabai and Dhingra [50] using superposition to efficiently handle reliability constraints in RBTO problems with uncertainty in loading using the BESO method. Recently, topology optimization of continuum structures under probabilistic and fuzzy loads is addressed by Liu et al. [51] using BESO method; in particular, the uncertainty of input data is described using a cloud model that permits to transform the uncertain topology optimization problem into a deterministic one with multiple load cases.

In this work, an ETO method driven by an optimality criterion is proposed for addressing the TOUU problem. This proposal includes some of the ingredients of the iso-XFEM method [19]; in particular, the use of implicit boundary representation by iso-contours, to control the shape and topology variations during the optimization process, and the extended finite element method (XFEM), to improve the accuracy of finite element solutions on the boundary of the design. The optimality criterion for the RTO problem is derived from the stochastic linear elasticity formulation in its weak form using a continuous adjoint method without being limited by the discretization method used for the physical and the stochastic domains. The ETO method uses an iterative approach to gradually add and/or remove material based on the iso-contours of the optimality criterion. The proposal permits to handle loading and material uncertainties modeled by different probability distributions and random field. To address the increment of dimensionality induced by the random field, an anisotropic sparse grid stochastic collocation method is used for the efficient computation of the multidimensional integrals over the random domain. Compared to density-based and level-set methods addressing TOUU problems, the proposal requires neither an initialization of the boundary nor any regularization parameter, and it provides smooth and clearly defined boundaries. Another important advantage of the proposal is that it provides optimal solutions for different volume fractions during the optimization process, which enables to efficiently find a trade-off between performance and robustness for different volume fractions during the topology optimization.

The remainder of the paper is organized as follows. The basis and theoretical background of TOUU problem and the RTO formulation are briefly reviewed in section 2 . Section 3 presents the adaptive sparse-grid stochastic collocation method used for uncertainty propagation and the efficient computation of the multidimensional integrals over the random domain required by the RTO formulation. The optimality criterion, used by the proposal to reach an optimal solution, is derived for the RTO problem in section 4 . Section 5 presents the proposed ETO algorithm driven by an optimality criterion to address the RTO problem. Section 6 is devoted to the numerical experiments used for validating the proposed method. Finally, section 7 presents the conclusion of the proposed ETO method for RTO problems. 


\section{Topology optimization under uncertainty (TOUU)}

The mathematical basis and fundamentals of TOUU problems and the specific formulation of RTO, addressed in this work, are presented below.

Let $(\Omega, \mathcal{F}, \mathbb{P})$ be a complete probability space, and let $D \subset$ $\mathbb{R}^{d}(d=2$ or $d=3)$ be a bounded Lipschitz domain whose boundary is decomposed into three disjoint parts $\partial D=\Gamma_{D} \cup$ $\Gamma_{N} \cup \Gamma_{0}$. Consider the linearized elasticity system under random input data

$$
\left\{\begin{array}{rlrl}
-\nabla \cdot \sigma(u(x, \omega))= & b(x, \omega) & & \text { in } D \times \Omega \\
u(x, \omega)= & \bar{u} & \text { in } \Gamma_{D} \times \Omega \\
\sigma(u(x, \omega)) \cdot n= & \bar{t}(x, \omega) & \text { in } \Gamma_{N} \times \Omega \\
\sigma(u(x, \omega)) \cdot n= & 0 & \text { in } \Gamma_{0} \times \Omega
\end{array}\right.
$$

where $x$ is the spatial variable, $\omega \in \Omega$ are the random events, $\sigma$ is the Cauchy stress tensor, $b$ and $\bar{t}$ are the body and surface forces, $\bar{u}$ is the prescribed displacement field, and $n$ is the unit outward normal vector to $\partial D$. The stress tensor $\sigma$ and the symmetric gradient of the displacement field $\varepsilon$ are related by means of the following constitutive equation

$$
\mathbb{C}(x, \omega): \varepsilon(x, \omega)=\sigma(x, \omega),
$$

where $\mathbb{C}$ represents the fourth order constitutive tensor and ":" operator is the double dot product of two tensors. Notice that compared to its deterministic counterpart, the body and surface forces and the constitutive tensor depend on a spatial variable $x$ and on a random event $\omega \in \Omega$. By using an Eulerian approach, the structural boundary $\partial O$ splits the domain $D$ into two different subdomains $O \subseteq D$ and $O^{c} \subseteq D$, such that $O \cup O^{c}=D$ and $O \cap O^{c}=\emptyset$. This subdivision into subdomains can be represented by means of a characteristic function $\chi$ such that

$$
\chi(x)=\left\{\begin{array}{ll}
1 & x \in O \\
0 & x \notin O
\end{array} .\right.
$$

This allows us to rewrite the constitutive tensor equation as

$$
\mathbb{C}(x, \omega)=\chi \mathbb{C}^{+}(x, \omega)+(1-\chi) \mathbb{C}^{-}(x, \omega),
$$

where $\mathbb{C}^{+}$and $\mathbb{C}^{-}$are the fouth order constitutive tensors of a stiff and a soft material respectively.

The TOUU problem is then formulated as the minimization of the structural compliance under random input data subjected to the maximum material allowed, as follows

$$
\begin{array}{cl}
\min _{\chi \in D_{L}} & J(\chi, \omega)=\int_{D} b u d x+\int_{\Gamma_{N}} \bar{t} u d s \\
\text { s. t. : } & a(\chi, u, v, \omega)=l(v, \omega) \quad \forall v \in V_{D}, \forall \omega \in \Omega \\
& D_{L}=\left\{\chi \in L^{\infty}(D,\{0,1\}),|O| \leq L|D|\right\}
\end{array}
$$

where $V_{D}=\left\{v \in H^{1}(D)^{d}:\left.v\right|_{\Gamma_{N}}=0\right\}, D_{L}$ is the feasible domain restricted to a volume constraint on $O$ denoted as a fraction $0<$ $L<1$ of the domain $D$, and $a(\chi, \cdot, \cdot, \cdot)$ and $l(\cdot, \cdot, \cdot)$ are the bilinear form and the right hand side of (1) obtained taking the weak form as follows

$$
\begin{aligned}
a(\chi, u, v, \omega) & =\int_{D} \sigma(u(x, \omega)): \varepsilon(v(x, \omega)) d x \\
l(v, \omega) & =\int_{D} b v d x+\int_{\Gamma_{N}} \bar{t} v d s .
\end{aligned}
$$

The general formulation of the TOUU problem (5) provides a different solution for each realization of the random event. The random functional should be transformed into a deterministic one to address the optimization problem using conventional optimization algorithms. Different formulations have been proposed in the literature depending on how the uncertainty is incorporated in the formulation. In this work, the formulation is focused on the RTO problem, which aims to find designs less sensitive to variations in the design variables and the input parameters. This is normally done by formulating the RTO problem as a two-objective optimization problem where the expected value and standard deviation of the compliance are considered as a measure of structural robustness.

The use of the weighted approach both in the structural optimization context [52, 53] and the RTO one [43, 38, 37] is extensive. The weighted sum method [54] incorporates a priori articulation of preferences by selecting weights, which are used to scalarize the multi-objective problem into a single-objective one. The solution of the single-objective problem allows the algorithm to determine a solution that presumably reflects such preferences. It is important to notice that the weighting coefficients do not reflect the relative importance of the objectives in the proportional sense, but they are only parameters varied to locate the non-dominated solution points. One can argue that the weights represent the trade-off between objectives, and thus maintaining the original unit of the objectives might be advantageous. An important recommendation adopted in this work is the use of unrestricted positive weights when articulating wellunderstood preferences [55].

It is well known that the major drawbacks of the weighted sum approach are its inability to capture solutions that lie on non-convex portions of the Pareto-optimal fronts [56] and the non-uniform distribution of points in the Pareto optimal set provided by the method with a consistent change in the weights. The former is due to the weighted sum utility function is only a linear approximation (in the criteria space) of the preference function [56], whereas the latter is attributed to the difficulty to set weights to indicate the relative importance of objectives. The systematic weight assignment is addressed by ranking methods in Multiple Objective Decision Making (MODM) [57] by ordering the objective functions by importance; in particular, the least important objective receives a positive weight and the objectives that are considered more important receive weight with consistent increments. A similar approach is commonly used in the RTO context to show the trade-off between the expected value and standard deviation of the compliance. Despite 
the drawbacks mentioned, the simplicity of the weighted approach has made it one of the most popular methods used to scalarize multi-objective problems.

The RTO problem is then formulated as follows

$$
\begin{array}{ll}
\min _{\chi \in D_{L}} & \left.J_{R}(\chi)=\mathbb{E}[J(\chi, \omega)]+\alpha \sqrt{\operatorname{Var}[J(\chi, \omega)}\right] \\
\text { s. t. : } & \int_{\Omega} a(\chi, u, v, \omega) d \mathbb{P}(\omega)=\int_{\Omega} l(v, \omega) d \mathbb{P}(\omega) \\
& \forall v \in L_{\mathbb{P}}^{2}\left(\Omega, V_{D}\right), \\
& D_{L}=\left\{\chi \in L^{\infty}(D,\{0,1\}),|O| \leq L|D|\right\},
\end{array}
$$

where $\mathbb{E}[\cdot]$ denotes the expectation operator, $\operatorname{Var}[\cdot]$ denotes the variance operator, and $\alpha \geq 0$ is a weight parameter to indicate the number of standard deviations from the mean to be considered. Increasing the parameter $\alpha$ has the effect of tightening the constraint on variability [38]. This is conceptually equivalent, as seen in [58], to increasing the robustness by adding penalty terms to constraints which include the tolerances of the design variables. In this probabilistic setting, the mean and the variance of the performance function are obtained as follows

$$
\begin{aligned}
\mathbb{E}[J(\chi, \omega)] & =\int_{\Omega} J(\chi, \omega) d \mathbb{P}(\omega), \\
\operatorname{Var}[J(\chi, \omega)] & =\int_{\Omega} J^{2}(\chi, \omega) d \mathbb{P}(\omega) \\
& -\left(\int_{\Omega} J(\chi, \omega) d \mathbb{P}(\omega)\right)^{2} .
\end{aligned}
$$

In practice, these multi-dimensional integrals cannot be evaluated analytically, and their numerical computation becomes computationally intractable as the dimension of the random domain increases. This issue has motivated the development of efficient methods to address the problem, such as dimension reduction methods and sparse grid collocation methods. In this work, due to the smoothness of the solution of the elasticity system an anisotropic non-intrusive sparse grid stochastic collocation method is used to evaluate the integrals in the random domain.

\section{Anisotropic non-intrusive sparse grid method}

The random state equation (1) may include uncertainties with spatial variations, such as material properties or distributed loads. These uncertainties are characterized by random fields. Let $z(x, \omega): D \times \Omega \longrightarrow \mathbb{R}$ be a random field, which is a function of the spatial coordinate $x$ and an element $\omega$ of the sample space. This random field is completely characterized by an infinite number of random variables, which is computationally intractable. To address this issue, the Karhunen-Loève (K-L) expansion [59] can be used to reduce the infinite-dimensional stochastic space to a finite-dimensional stochastic one. A truncated K-L expansion of the random field $z(x, \omega)$ can be written as

$$
z(x, \omega) \cong \bar{z}(x)+\sum_{i=1}^{N} \sqrt{\gamma_{i}} \phi_{i}(x) y_{i}(\omega),
$$

where $\bar{z}(x)$ is the mean function, $\gamma_{i}$ and $\phi_{i}$ are the eigenvalues and eigenvectors, respectively, of the compact and self-adjoint operator

$$
\psi \mapsto \int_{D} C\left(x, x^{\prime}\right) \psi\left(x^{\prime}\right) d x^{\prime}, \quad \psi \in L^{2}(D),
$$

and $y_{i}(\omega)$ are the terms of a vector of independent random variables.

The number of terms of the truncated K-L expansion (10) is related to the decay rate of its eigenvalues. Thus, the number of terms required to reach a specified error threshold depends on the correlation function of the process. Thus, a large number of terms is needed to approximate the random field with reasonable accuracy for a relatively small correlation length. In this case, the use of full tensor product rules for solving the multi-dimensional integrals in (9) can lead to an unaffordable computational problem. To overcome this difficulty, the isotropic sparse grid approaches [60] were proposed to reduce the number of collocation points keeping the level of accuracy for problems whose random variables weigh equally in the solution. However, the convergence rate is deteriorated for highly anisotropic problems [61], which is the resulting case from $\mathrm{K}$ $\mathrm{L}$ expansions. In this case, an anisotropic sparse tensor product can drastically reduce the computational cost while providing highly accurate solutions.

The numerical approximation in the random domain is performed using the adaptive anisotropic sparse grid collocation method described below. For an integer $\ell \in \mathbb{N}^{+}$, called the level, and a vector of weights for the different stochastic directions $\boldsymbol{\beta}=\left(\beta_{1}, \beta_{2}, \cdots, \beta_{N}\right)$, consider the index set

$\mathbf{X}_{\beta}(\ell, N)=\left\{\mathbf{i}=\left(i_{1}, \cdots, i_{N}\right) \in \mathbb{N}_{+}^{N}, \mathbf{i} \geq \mathbf{1}: \sum_{n=1}^{N}\left(i_{n}-1\right) \beta_{n} \leq \ell \underline{\beta}\right\}$,

with $\beta:=\min _{1 \leq n \leq N} \beta_{n}$. Some examples of isotropic and anisotropic Smolyak grids are illustrated in Figure 1. Similarly to the isotropic Smolyak quadrature rule [60], the anisotropic Smolyak quadrature formula applied to a generic function $f$ is given by

$$
\begin{aligned}
\mathscr{A}_{\beta}(\ell, N) f & =\sum_{\mathbf{i} \in \mathbf{X}_{\beta}(\ell, N)}\left(\Delta^{i_{1}} \otimes \cdots \otimes \Delta^{i_{N}}\right) f \\
& =\sum_{r_{1}=1}^{R_{i_{1}}} \cdots \sum_{r_{N}=1}^{R_{i_{N}}} f\left(y_{1}^{r_{1}}, \cdots, y_{N}^{r_{N}}\right) w_{1}^{r_{1}} \cdots w_{N}^{r_{N}},
\end{aligned}
$$

where $\Delta^{i_{n}}=Q^{i_{n}}-Q^{i_{n}-1}$, with $Q^{0}=0$, is a quadrature rule in which the coordinates $y_{n}^{r_{n}}$ of the nodes are those for the 1D quadrature formula $Q^{i_{n}}$ and their associated weights $w_{n}^{r_{n}}$ are the difference between those for the $i_{n}$ and $i_{n}-1$ levels. The number of collocation points in the $n$-th direction is denoted by $R_{i_{n}}$. The vector of weights $\boldsymbol{\beta}$ depends on the analyticity properties of the solution $u(x, y)$ with respect to $y$. For the case of Gaussian random variables $[62,63]$, the $n$-th term of the vector $\beta$ takes the following form 


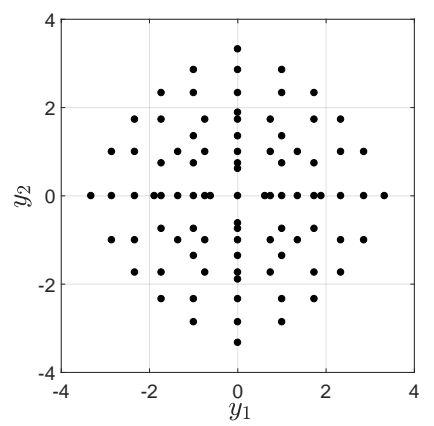

(a)

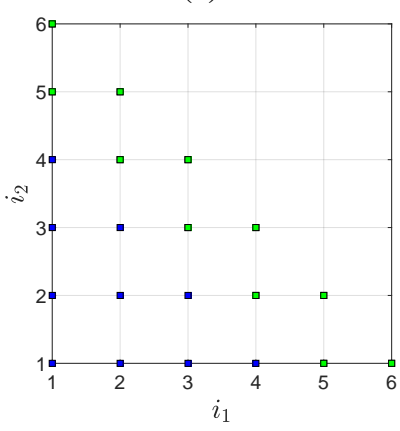

(c)

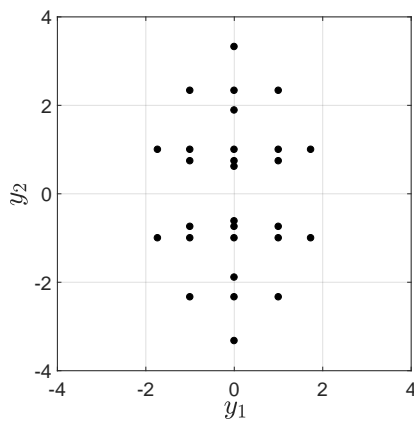

(b)

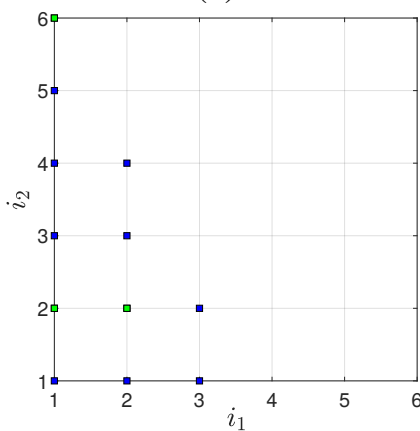

(d)
Figure 1: Smolyak grids for $\ell=5, N=2$ and Gaussian 1d quadrature: (a) sparse grid and (c) index set obtained by isotropic sparse tensor product with $g=[1,1]$, and (b) sparse grid and (d) index set obtained by anisotropic sparse tensor product with $g=[1,0.5]$.

$$
\beta_{n}=\frac{1}{2 \sqrt{2} \sqrt{\gamma_{n}}\left\|\phi_{n}\right\|_{L^{\infty}(D)}},
$$

with $\left(\gamma_{n}, \phi_{n}(x)\right)$ the eigenpair in the K-L expansion.

The level $\ell$ of the quadrature rule is adaptively chosen as to comply with a prescribed level of accuracy $0<\varepsilon<<1$. This is done as follows:

1. Calculate the vector of weights $\beta$ according to (13).

2. Initialize the level $\ell=1$ and a positive, large enough, integer $\bar{\ell}$.

3. Determine the discrete numerical approximation of the performance function $\mathscr{A}_{\beta}(\ell, N) J(\chi, \omega)$. The compliance $J(\chi, \omega)$, given by (5), is obtained solving the state equation $a\left(\chi, u \chi, v, y^{k}\right)=l\left(v, y^{k}\right)$ at the stochastic nodes $y^{k}$, which come from the quadrature rule (12).

4. Calculate the norm over $L^{2}(D)$ of the mean and the variance of $J(\chi, \omega)$ denoted by

$$
E_{\beta, \ell, N}=\left\|\mathscr{A}_{\beta}(\ell, N) J(\chi, \omega)\right\|_{L^{2}(D)}^{2}
$$

and

$$
V_{\beta, \ell, N}=\left\|\mathscr{A}_{\beta}(\ell, N) J^{2}(\chi, \omega)-\mathscr{A}_{\beta}(\ell, N) J(\chi, \omega)^{2}\right\|_{L^{2}(D)}^{2} .
$$

5. Calculate the numerical approximation of an enriched solution given by

$$
\mathscr{A}_{\hat{\beta}}(\bar{\ell}+1, N) J(\chi, \omega) \text {, with } \hat{\beta}_{n}=\left(\frac{I_{n}(\boldsymbol{\beta}, \bar{\ell})-1}{I_{n}(\boldsymbol{\beta}, \bar{\ell})}\right)\left(\frac{\beta_{n}}{\underline{\beta}}\right) \text {, }
$$

where the maximum index in each direction $I_{n}(\beta, \bar{\ell})=$ $\max _{i \in X_{\beta}(\bar{\ell}, N)}\left\{i_{n}\right\}$ is introduced.

6. Compute the norm over $L^{2}(D)$ of the mean and the variance of the enriched solution as follows

$$
E_{\hat{\boldsymbol{\beta}}, \bar{\ell}, N}=\left\|\mathscr{A}_{\hat{\boldsymbol{\beta}}}(\bar{\ell}+1, N) J(\chi, \omega)\right\|_{L^{2}(D)}^{2}
$$

and

$$
\begin{aligned}
& V_{\hat{\beta}, \bar{\ell}, N}= \\
& \left\|\mathscr{A}_{\hat{\beta}}(\bar{\ell}+1, N) J^{2}(\chi, \omega)-\mathscr{A}_{\hat{\beta}}(\bar{\ell}+1, N) J(\chi, \omega)^{2}\right\|_{L^{2}(D)}^{2}
\end{aligned}
$$

7. Finally, the level $\ell$ is increased linearly (from $\ell=1$ to $\left.\ell=\ell_{\text {opt }} \leq \bar{\ell}\right)$ up to the stopping criterion

$$
\max \left\{\frac{\left|E_{\boldsymbol{\beta}, \ell, N}-E_{\hat{\boldsymbol{\beta}, \bar{\ell}, N}}\right|}{E_{\hat{\boldsymbol{\beta}}, \bar{\ell}, N}} \quad, \quad \frac{\left|V_{\boldsymbol{\beta}, \ell, N}-V_{\hat{\boldsymbol{\beta}}, \bar{\ell}, N}\right|}{V_{\hat{\boldsymbol{\beta}}, \bar{\ell}, N}}\right\} \leq \varepsilon
$$

is satisfied.

\section{Optimality criterion for Robust Topology Optimization}

Following the Céa's classical Lagrange method [64], the constrained minimization problem (8) is transformed into an unconstrained minimization problem using the augmented Lagrangian function

$$
\begin{aligned}
\mathcal{L}(\chi, u, p) & =J_{R}(\chi, u)+\int_{\Omega} a\left(\chi_{O}, u, p, \omega\right) d \mathbb{P}(\omega) \\
& -\int_{\Omega} l(p, \omega) d \mathbb{P}(\omega)+\lambda\left(\int_{O} \chi d x-L|D|\right),
\end{aligned}
$$

where $p \in L_{\mathbb{P}}^{2}\left(\Omega, V_{D}\right)$ is the adjoint state and $\lambda$ is the Lagrange multiplier used to enforce the volume constraint at convergence. Notice that the augmented Lagrangian function is defined for any characteristic function $\chi \in D_{L}$, and for any $u, p \in L_{\mathbb{P}}^{2}\left(\Omega, V_{D}\right)$, so that the variables are independent.

For a fixed $\chi$, we search for the saddle points $(u, p) \in$ $L_{\mathbb{P}}^{2}\left(\Omega, V_{D}\right)$ of $\mathcal{L}(\chi, \cdot, \cdot)$. First, we take the derivative of $\mathcal{L}$ with respect to $\hat{p}$ in the direction $q \in L_{\mathbb{P}}^{2}\left(\Omega, V_{D}\right)$. After integrating by parts and equating to zero gives

$$
\begin{aligned}
\frac{\partial \mathcal{L}\left(\chi_{, u, p)}\right.}{\hat{p}}(q) & =\int_{\Omega} \int_{D} \sigma(u): \varepsilon(q) d x d \mathbb{P}(\omega) \\
& -\int_{\Omega} \int_{D} b q d x d \mathbb{P}(\omega)-\int_{\Omega} \int_{\Gamma_{N}} \bar{t} q d s d \mathbb{P}(\omega)=0,
\end{aligned}
$$

where the weak form of the state equation is recovered, whose total sum is zero. Similarly, taking the derivative of $\mathcal{L}$ with respect to $\hat{u}$ in the direction $v \in L_{\mathbb{P}}^{2}\left(\Omega, V_{D}\right)$ and integrating by parts the following adjoint equation is obtained

$$
\begin{aligned}
\frac{\partial \mathcal{L}\left(\chi_{, u, p)}\right.}{\hat{u}}(v) & =\int_{\Omega} \int_{D} \sigma(p): \varepsilon(v) d x d \mathbb{P}(\omega) \\
& -\int_{\Omega} \int_{D}\left(1+\frac{\alpha}{\operatorname{Std}[J]} J(\chi, \omega)-\frac{\alpha \mathbb{E}[J]}{\operatorname{Std}[J]}\right) b v d x d \mathbb{P}(\omega) \\
& -\int_{\Omega} \int_{\Gamma_{N}}\left(1+\frac{\alpha}{\operatorname{Std}[J]} J(\chi, \omega)-\frac{\alpha \mathbb{E}[J]}{\operatorname{Std}[J]}\right) \bar{t} v d s d \mathbb{P}(\omega) \\
& =0 .
\end{aligned}
$$

The shape derivative of the Lagrangian functional is given by 


$$
\begin{aligned}
\frac{\partial \mathcal{L}(\chi, u, p)}{\partial \mathcal{X}}(\theta) & =\int_{\Gamma_{0}} \theta \cdot n\left(\int_{\Omega} b \cdot u+\alpha \frac{1}{\operatorname{Std}[J]}(J(\chi, \omega) b \cdot u)\right. \\
& \left.-\alpha \frac{\mathbb{E}[J]}{\operatorname{Std}[J]} b \cdot u-b \cdot p+\sigma(u): \varepsilon(p) d \mathbb{P}(\omega)+\lambda\right) d s \\
& =0,
\end{aligned}
$$

where the following optimality criterion for the RTO problem can be derived

$$
\begin{aligned}
\left.\Phi(\chi, x)\right|_{x \in \partial O} & =\int_{\Omega}\left\{-b \cdot u-\alpha \frac{1}{\operatorname{Std}[J]}(J(\chi, \omega) b \cdot u)\right. \\
& \left.+\alpha \frac{\mathbb{E}[J]}{\operatorname{Std}[J]} b \cdot u+b \cdot p-\sigma(u): \varepsilon(p)\right\} d \mathbb{P}(\omega) \\
& =\lambda
\end{aligned}
$$

That is, the optimal structural design should satisfy the condition $\Phi(\chi, x)=\lambda$ in the boundary of the design. Such an optimal criterion can be used to evolve the search for the optimum design using evolutionary methods. This is done using an iterative approach to adaptively update the Lagrange multiplier $\lambda \in \mathbb{R}$ based on the optimal criterion until the design satisfying the final amount of material is reached.

Assuming that body forces are not present in our problem, i.e. $b=0$ from (17) to (20), and given a set of $N_{s g}$ sparse grid nodes $\left(y_{1}, \ldots, y_{N_{s g}}\right)$ and weights $\left(w_{1}, \ldots, w_{N_{s g}}\right)$, the criterion $\Phi(\chi, x)$ can be approximated as

$$
\Phi(\chi, x) \cong \sum_{k=1}^{N_{s g}}\left(\sigma\left(u\left(x, y_{k}\right)\right): \varepsilon\left(p\left(x, y_{k}\right)\right)\right) w_{k}
$$

where $p\left(x, y_{k}\right)$ is the adjoint state at the collocation point $y_{k}$ obtained solving the system

$$
\begin{aligned}
& \int_{D} \sigma\left(p\left(x, y_{k}\right)\right): \varepsilon(v) d x= \\
& \int_{\Gamma_{N}}\left(1+\frac{\alpha}{\operatorname{Std}[J]} J\left(\chi, y_{k}\right)-\frac{\alpha \mathbb{E}[J]}{\operatorname{Std}[J]}\right) \bar{t}\left(x, y_{k}\right) v d s
\end{aligned}
$$

for all $v \in V_{D}$.

\section{Evolutionary method for updating the optimal criterion}

The proposed method makes use of the optimality criterion derived in the previous section to obtain optimal shapes that satisfy such a criterion in the boundary of the structural design. We can consider the proposal as a variant of ESO method that gradually adds and/or removes material depending on the shape and distribution of the optimality criterion in the design. This approach is similar to the method used in other works using strain energy density or von Mises stress [19] to evolve the structural design. The method defines the structural boundary by the intersection of the structural performance distribution with a minimum level of performance, which is increased during the optimization process. Compared to traditional ETO methods, such $\mathrm{ESO} / \mathrm{BESO}$, the method driven by an optimal criterion provides solutions with a smooth boundary representation, which facilitates the topology interpretation.

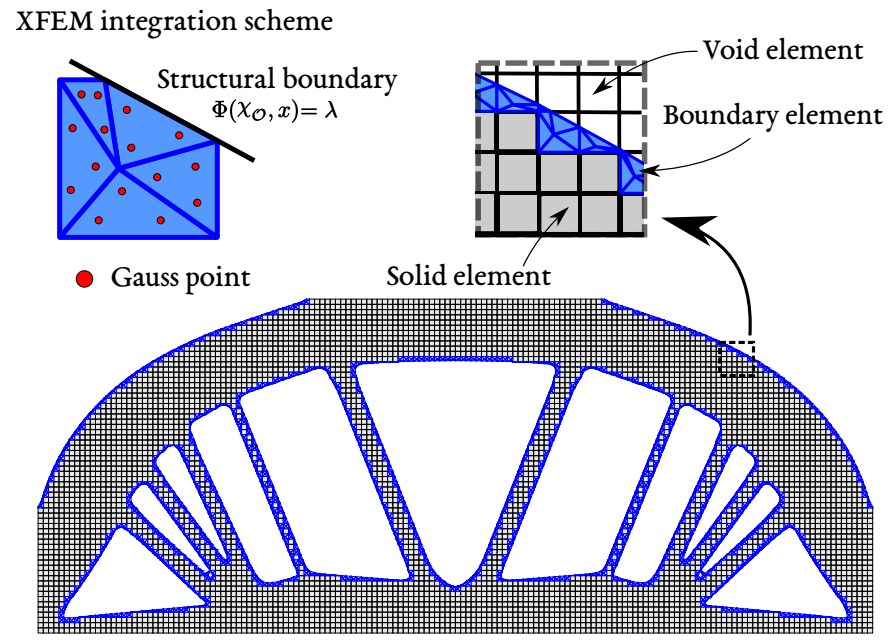

Figure 2: Classification of elements using fixed grid approach and XFEM integration scheme for boundary elements.

In this work, the iso-XFEM approach [19] is adopted to gradually update the Lagrange multiplier $\lambda$, which defines the structural boundary. This is done by superimposing the structural domain $O$ over a regular grid of rectangular equally sized elements, as shown in Figure 2. Three types of elements are defined: elements located inside $O$ (solid elements), elements located outside $O$ (void elements), and boundary intersected elements (boundary elements). The integration is performed using the XFEM approach. That is, the solid and void elements are integrated using conventional finite element method (FEM), whereas boundary elements are integrated on the corresponding solid sub-domain. Figure 2 shows how the solid sub-domain of the boundary elements is partitioned into triangles, which are integrated using three Gauss quadrature points. The element stiffness matrix of the boundary elements is obtained adding the contribution of each triangle.

The proposed ETO method is summarized into the following steps:

1. Define the RTO problem, including the regular grid of finite elements, boundary conditions, solid and void material properties, optimizable and non-optimizable regions, and uncertain variables.

2. Define the target volume $V^{*}$, an evolutionary volume ratio $(E R)$ and the allowable volume change $\Delta V$ for stabilization purposes.

3. Calculate the approximate distribution $\Phi(\chi, x)^{(i)}$ of (21) using XFEM for solving the state and adjoint state equations at each stochastic collocation point at the current iteration of the optimization approach.

4. Average the criterion with the values of previous iterations $\Phi(\chi, x)^{(i)}=\frac{\Phi(\chi, x)^{(i)}+\Phi(\chi, x)^{(i-1)}}{2}$ to stabilize convergence [65].

5. Calculate the target volume for the current iteration following $V^{(i)}=\max \left\{V^{(i-1)}(1-E R), V^{*}\right\}$. Once the objective volume is reached, the volume will be kept constant for the remaining iteration. 
6. Calculate the value of the Lagrange multiplier $\lambda^{(i)}$ intersecting with $\Phi(\chi, x)^{(i)}$ that gives the target volume at the current iteration $V^{(i)}$. This is done using a bisection method.

7. Extract the boundary of the design $\partial O$ and assign solid and void material properties according to

$$
\Phi(\chi, x)^{(i)} \begin{cases}>\lambda^{(i)} & x \in O \\ =\lambda^{(i)} & x \in \partial O . \\ <\lambda^{(i)} & x \notin O\end{cases}
$$

8. Perform a stabilization loop to evolve the structural boundary to the corresponding iso-value $\lambda^{(i)}$. This is an iterative process of reanalysis and material distribution until the volume change in successive iterations is lower than the empiric value $\Delta V$.

9. Repeat from 3 to 8 to gradually reduce the volume $V^{(i)}$ until the target volume $V^{*}$ satisfying the optimal criterion is obtained.

Notice that the topology optimization problem in elasticity is known to be ill-posed since there is not always existence of solutions in the set of admissible shapes [66] and there is usually non-uniqueness of the solution [67]. The existence of, at least, one solution in the set of admissible shapes can be proven under certain constraints [42]. The practical consequence is that numerical methods may exhibit numerical instabilities and the optimal design depends on several factors, such as the initial design and the parameters used for the optimization problem, to name but a few. The strategy used to update the Lagrange multiplier is mesh dependent, which is typically the case for ETO methods and element based topology optimization methods. This issue has been studied in a deterministic settings in earlier investigations for BESO [68, 65] and for iso-XFEM methods [19]. Such works indicate that pseudo mesh-independent topologies can be obtained using filtering strategies. Additionally, these strategies may decrease the complexity of the convergence solutions at the cost of degrading the performance of the optimal topology.

\section{Numerical Experiments}

The effectiveness of the proposed development for RTO of continuous structures is evaluated using three benchmarks commonly used in the TOUU literature. The first two problems ("beam-to-cantilever" [31, 37, 42] and "carrier plate" [37, 35]) aim at optimizing the design domain under uncertain loading characterized by different probability distributions and random fields. The last problem ("simply supported beam" $[37,35]$ ) is concerned with the optimization problems combining loading and material uncertainties. These experiments aim at showing that the proposed development is able to deal with different probability distributions as well as random fields. All the benchmarks make use of a fixed grid of $\mathbb{Q}_{1}$ quadrangular plane stress finite elements with Poisson's ratio $v=0.3$ and Young's modulus $E_{\text {solid }}=1$ and $E_{\text {void }}=1 \mathrm{e}-5$ for solid and void material respectively.

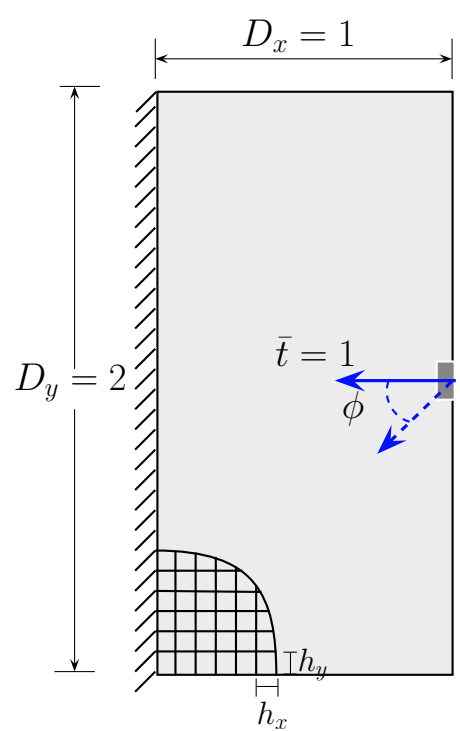

(a)

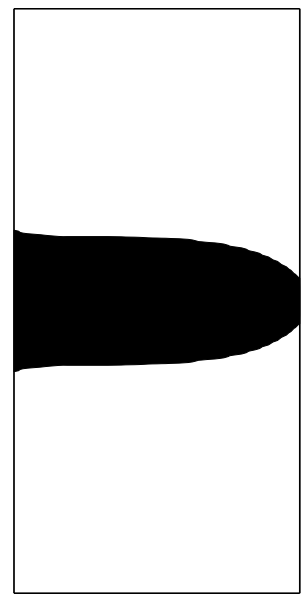

(b)
Figure 3: The beam-to-cantilever problem: (a) the design domain and boundary conditions, and (b) the deterministic optimal design.

\subsection{Beam-to-cantilever problem}

The beam-to-cantilever problem consists in the topology optimization of a two-dimensional cantilever under uncertain loading conditions. Such a benchmark is widely used [31, 37, 42] to show the effect of uncertain loading in the robust optimal design. The left edge of the cantilever is anchored and a unitary force with uncertainty in direction, centered in the horizontal line, is applied in the middle of the right edge. The design domain is a $1 \times 2$ rectangle tessellated using a $64 \times 128$ quadrangular mesh $\left(h_{1}=h_{2}=0.0156\right)$. The uncertain loading, the boundary conditions, and the tessellation of the design domain are shown in Figure 3(a), whereas the topology design using a deterministic approach is shown in Figure 3(b).

The configuration of the numerical resolution of the RTO problem is as follows. The target volume $V^{*}$ is set to the $20 \%$ of the initial design domain. The direction $\phi$ of the unit-load follows a Gaussian distribution centered at the horizontal line with mean $\mu_{\phi}=0$ and standard deviation $\sigma_{\phi}=\pi / 12$. Two different probability distributions of $\phi$ are evaluated to study the effect of the skewness on the robust topology design. In particular, Gaussian and Gumbel probabilistic distributions are considered. Non-normal stochastic distributions are transformed into a Gaussian distribution, and then a Gauss-Hermite quadrature rule with a level $\ell=5$ is used to evaluate the integrals involved in the optimality criterion. The evolutionary volume ratio $E R=0.005$ and the allowable volume change $\Delta V=0.001$ are used in the experiments. The linear system of equations of elasticity is solved using a conjugate gradient method and a V-cycle geometric multigrid preconditioning using 6 levels, a damping factor for Jacobi smoothing $w=0.4$ and a tolerance tol $=1 \mathrm{e}-8$.

Figure 4 shows the robust optimized designs for the minimization of the functional cost $J_{R}$ with different values of $\alpha$. 


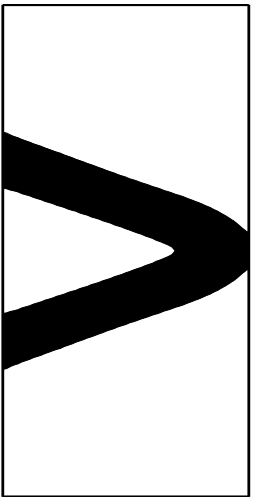

(a) $\alpha=0$

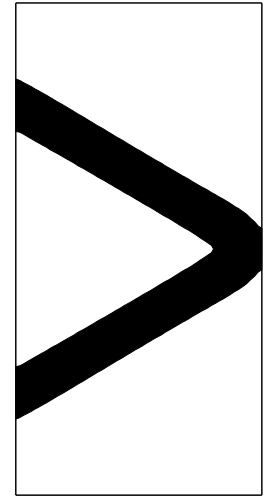

(d) $\alpha=3$

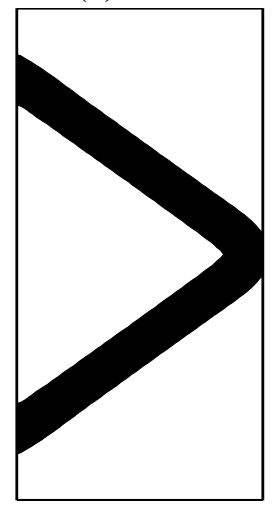

(g) $\alpha=6$

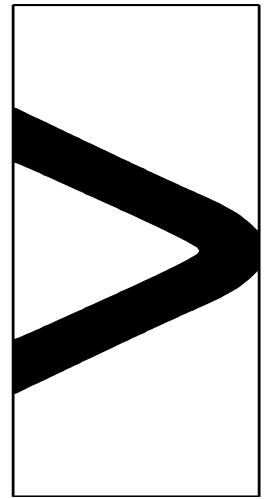

(b) $\alpha=1$

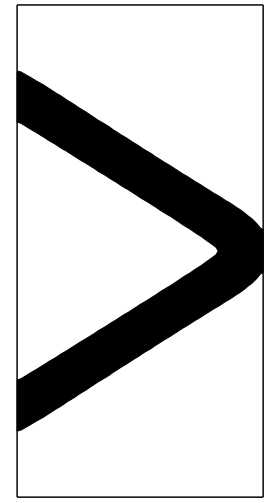

(e) $\alpha=4$

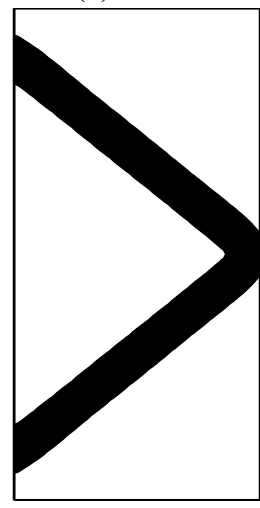

(h) $\alpha=8$

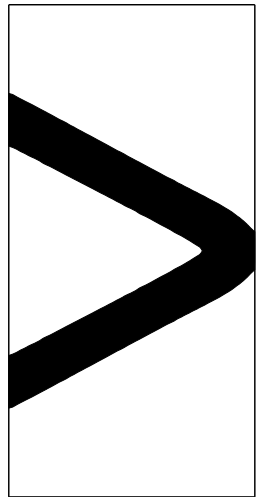

(c) $\alpha=2$

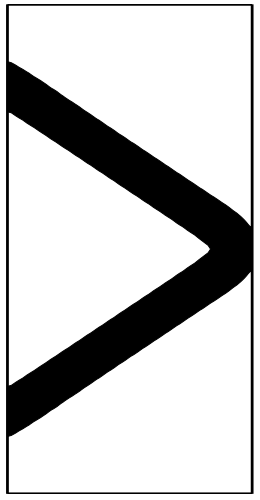

(f) $\alpha=5$

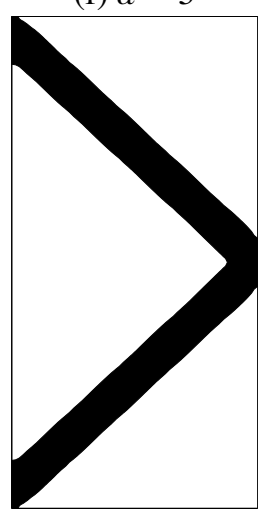

(i) $\alpha=10$
Figure 4: Robust optimized designs using different values of $\alpha$ for the beam-tocantilever problem with the direction of the unit-load $\phi \sim N(0, \pi / 12)$.
Table 1: Statistical moments of compliance and volume fraction for different values of $\alpha$ solving the beam-to-cantilever problem.

\begin{tabular}{c|ccc}
\hline$\alpha$ & $\mathbb{E}[J]$ & $\operatorname{Std}[J]$ & Vol. frac. \\
\hline Det. & 6.127 & 4.0741 & 0.199 \\
0 & 4.487 & 1.336 & 0.200 \\
1 & 4.642 & 0.939 & 0.200 \\
2 & 4.877 & 0.780 & 0.199 \\
3 & 5.160 & 0.658 & 0.200 \\
4 & 5.449 & 0.575 & 0.202 \\
5 & 5.716 & 0.512 & 0.200 \\
6 & 6.122 & 0.434 & 0.201 \\
8 & 7.033 & 0.310 & 0.201 \\
10 & 8.694 & 0.127 & 0.200 \\
\hline
\end{tabular}

The resulting robust designs incorporate stiffness in the vertical direction where the uncertain loading is introduced. The mean and standard deviation of compliance for deterministic and robust designs are shown in Table 1. One can observe that the standard deviation of all the robust designs is smaller than the one obtained for the deterministic design. This verifies the improvement of the robust designs in terms of robustness. In addition, the expected performance decreases as the weight of variance increases in the functional cost. This unveils the multi-objective nature of the RTO problem and the importance of finding a trade-off between structural robustness and expected structural performance. The resulting front of robust optimal solutions for the beam-to-cantilever problem, shown in Figure 6(a), provides the designer useful information for the decision-making process, where the preferences between conflicting objectives should be chosen.

The effect of the probability distribution used to model the uncertainty of the direction $\phi$ of the unit-load is shown in Figure 5 , where the density of probability is represented by the magnitude of arrows for the corresponding $\phi$. One can observe that the probability distribution used to model the uncertainty has a notable influence on the optimal design. We obtain a symmetric optimal design using a Gaussian distribution for modeling the uncertainty in the direction $\phi$ of the unit-load, whereas the resulting optimal design using a Gumbel distribution is asymmetric by thickening the cantilever members in the direction of the skew.

One of the advantages of the proposed approach is that it provides the designer with a set of optimal solutions at different volume fractions. That is, the intermediate designs with different amount of material used to reach the optimal design satisfying the volume constraint, are also optimal designs for the corresponding volume fraction. Such intermediate robust designs can be used to obtain a trade-off between performance and robustness for different volume fractions. Figure 7 shows the trade-off between performance and robustness for different volume fractions obtained during the RTO of the beamto-cantilever problem using the proposed method. This set of optimal solutions provides valuable and relevant information to support the designer decisions, permitting to find a trade-off between performance (stiffness of compliance) and robustness 
(standard deviation) for different volume fractions of material.

\subsection{Carrier plate under distributed uncertain load}

We use the carrier plate problem [37] to show the performance of the proposal for solving RTO problems using random fields to model uncertain loading. The boundary conditions and uncertain loading are shown in Figure 8(a). The design domain consists of a square of side length 10 . The domain is tessellated with a regular mesh of $128 \times 128 \mathbb{Q}_{1}$ elements. Three layers of elements at the top of the domain are defined as nonoptimizable to ensure the structure remains attached to the loading conditions. The structure is subjected to a uniformly vertically distributed load $\bar{t}_{2}=10$ acting at its top. The vertical load is perturbed with a random horizontal distributed load $\bar{t}_{1}(x, \omega)$ applied at its top edge. The random load magnitude is modeled as a Gaussian random field with zero mean and isotropic square exponential covariance function

$$
C\left(x, x^{\prime}\right)=\sigma^{2} \exp \left[-\sum_{i=1}^{2} \frac{\left(x_{i}-x_{i}^{\prime}\right)^{2}}{l^{2}}\right]
$$

where $x=\left(x_{1}, x_{2}\right), x^{\prime}=\left(x_{1}^{\prime}, x_{2}^{\prime}\right) \in \Gamma_{N}, \sigma=5$ is the standard deviation, and $l=1.5$ is the correlation length.

According to the decay rate of the eigenvalues, the K-L expansion is truncated at its fifth term to capture $60 \%$ of the energy field. Since the considered random variables $Y_{n}$ are Gaussian, a non-nested quadrature rule, whose collocation nodes $y^{k}$ are determined by the roots of Hermite polynomials, is used. The nodes and weights of the anisotropic sparse grid are computed adaptively as described above with $N=5, \bar{\ell}=7, \varepsilon=1 \mathrm{e}-2$ and $\ell_{\text {opt }}=3$. The convergence rate, shown in Figure 9, reveals a (sub)-exponential decay of the error as the number of points increases linearly. Both the level and the number of points of the anisotropic sparse grid method are governed by the error of the variance of the compliance, which has the slowest rate of convergence. By using 61 sparse grid nodes, errors of 2e-3 and $1.2 \mathrm{e}-12$ are achieved for the variance and the mean value of the compliance, respectively.

The target volume $V^{*}$ is set to the $35 \%$ of the initial design domain, and the evolutionary volume ratio $\mathrm{ER}=0.005$ and the allowable volume change $\Delta V=0.005$ are used for the experiments. The linear system of equations of elasticity is solved using a conjugate gradient method preconditioning and a V-cycle geometric multigrid preconditioning with 5 levels, a damping factor for Jacobi smoothing $\mathrm{w}=0.4$ and a tolerance of $1 e-8$.

Figure 10 shows the robust topology designs obtained increasing the value of the parameter $\alpha$. One can observe that the robust topology designs differ meaningfully compared to the deterministic optimal design shown in Figure 8(b). The robust designs show a larger span between supports compared to the deterministic counterpart. Besides, the increase in robustness is firstly achieved by means of topology modifications, increasing the redundancy by adding paths to transmit the load to the support, and then by thickening the members. We also can observe that the robust designs incorporate diagonal members to withstand the loading direction uncertainty. The expected value and the standard deviation of the compliance, detailed in Table 2, 


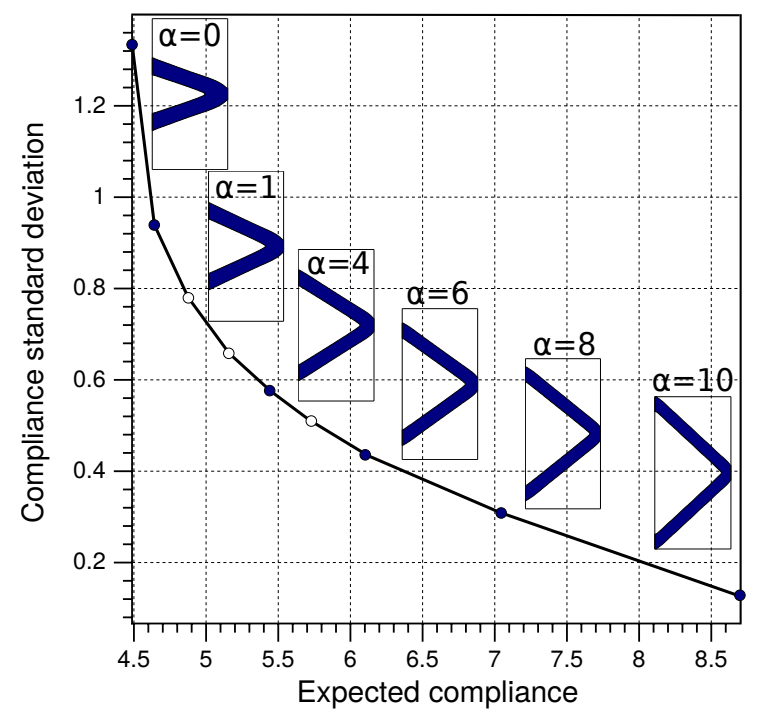

(a)

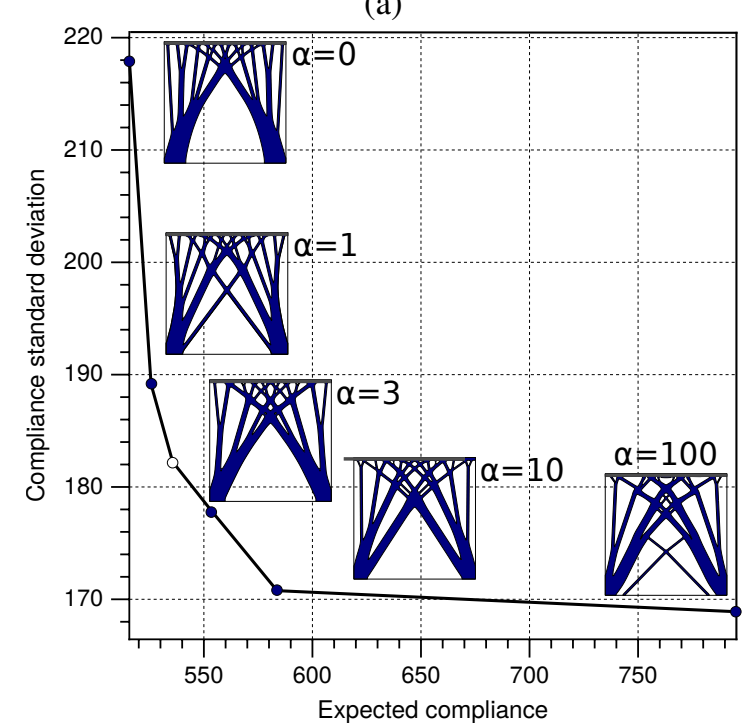

(b)

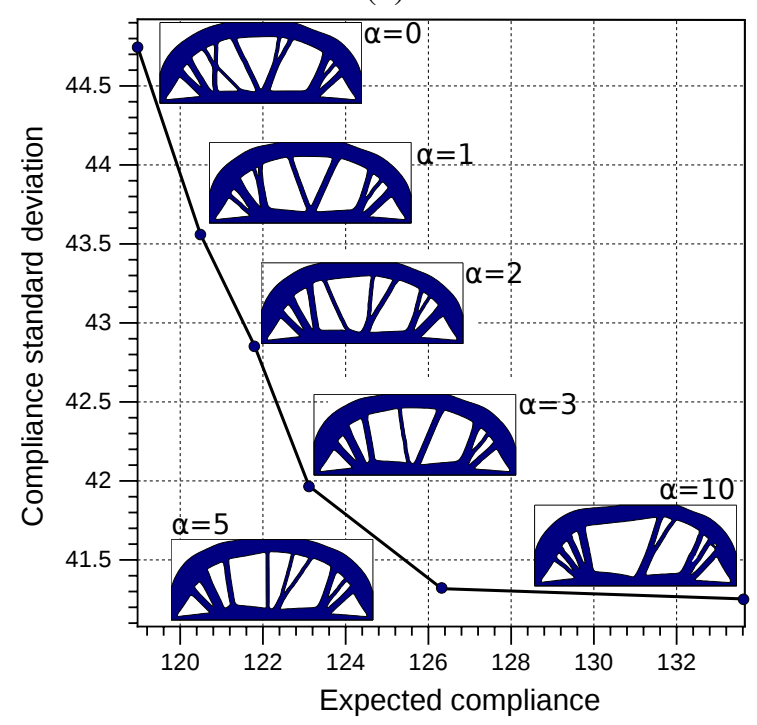

(c)

Figure 6: Fronts of robust optimal solutions for (a) the beam-to-cantilever problem, (b) the carrier plate design and (c) the simply supported beam.

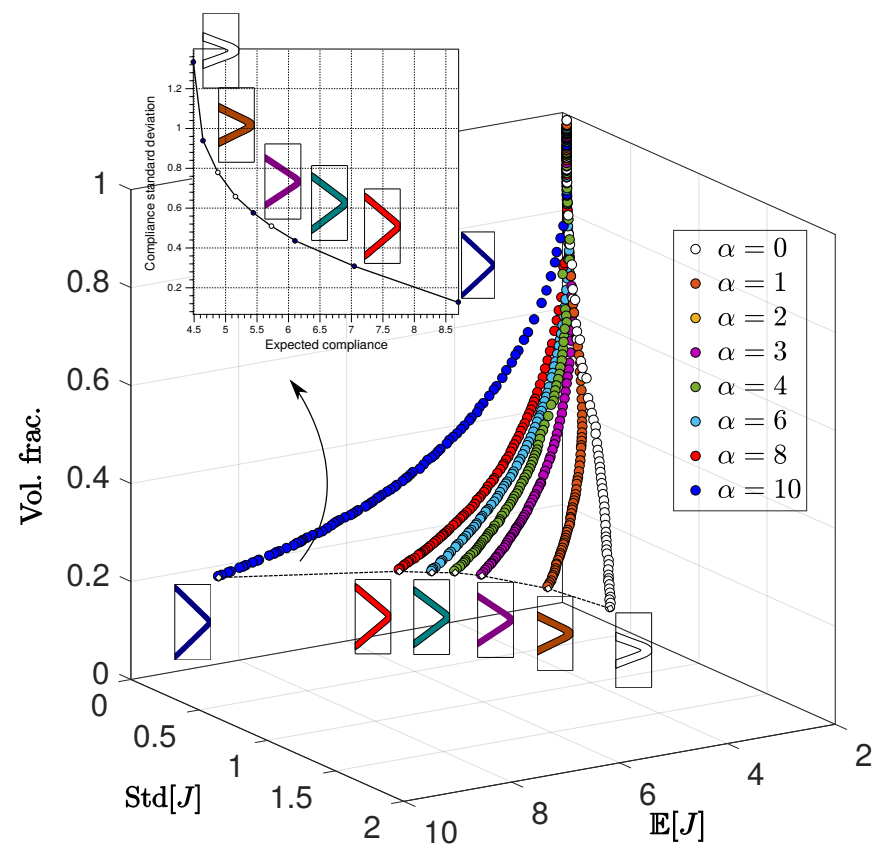

Figure 7: Trade-off between performance and robustness for different volume fractions for the beam-to-cantilever problem.

Table 2: Statistical moments of compliance and volume fraction for different values of $\alpha$ solving the carrier plate benchmark.

\begin{tabular}{c|ccc}
\hline$\alpha$ & $\mathbb{E}[J]$ & $\operatorname{Std}[J]$ & Vol. frac. \\
\hline Det. & 2821.8 & 3532.8 & 0.350 \\
0 & 515.658 & 218.052 & 0.349 \\
1 & 525.686 & 189.144 & 0.349 \\
2 & 535.601 & 182.245 & 0.349 \\
4 & 552.790 & 178.044 & 0.349 \\
10 & 583.721 & 170.770 & 0.349 \\
100 & 795.377 & 168.894 & 0.350 \\
\hline
\end{tabular}

show the improved performance of robust designs compared to their deterministic counterpart. Figure 6(b) shows the trade-off between performance and robustness for different values of the $\alpha$ parameter.

\subsection{Simply supported beam under loading and material uncer- tainties}

The simply supported beam experiment is used to evaluate the ability of the proposal to obtain optimal designs in RTO problems with combined loading and material uncertainties. Figure 11(a) shows the boundary conditions and the design domain, which is tessellated using a regular grid of 200x80 linear quadrangular elements. The structure is subjected to three uncertain vertically concentrated loads, whose moduli are characterized by three independent random variables. These random variables follow a Gaussian distribution with mean values $\mu_{\bar{t}_{1}}=\mu_{\bar{t}_{2}}=\mu_{\bar{t}_{3}}=1$ and standard deviations $\sigma_{\bar{t}_{1}}=0.5, \sigma_{\bar{t}_{2}}=0.1$ and $\sigma_{\bar{t}_{3}}=0.2$. The Young's modulus is modeled by a 2D lognormal random field with mean $\mu_{F}=1$ and standard deviation 


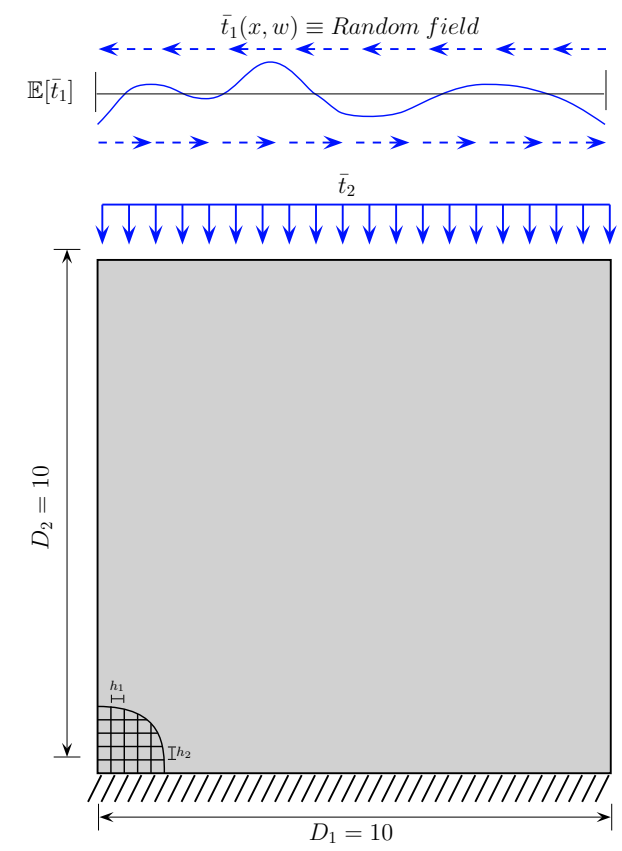

(a)

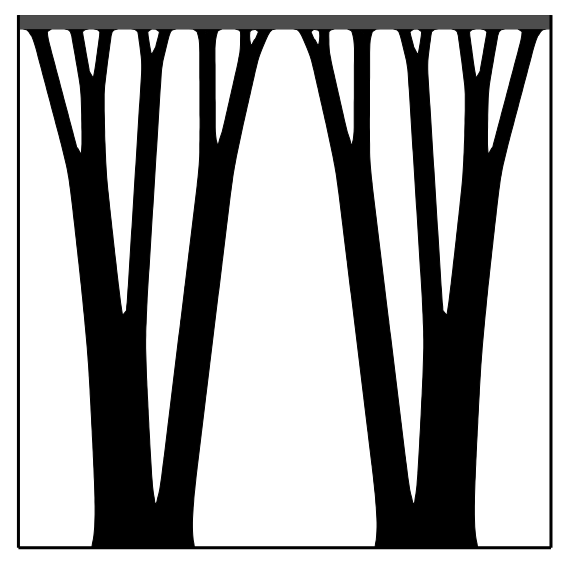

(b)

Figure 8: The carrier plate benchmark: (a) the design domain and boundary conditions, and (b) the deterministic optimal design.

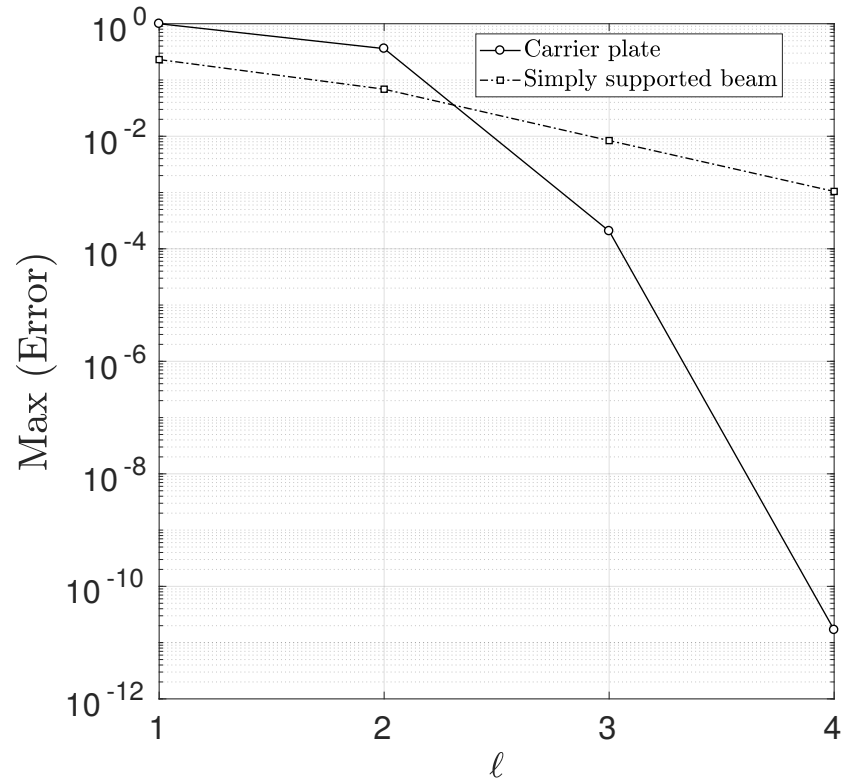

Figure 9: The rates of convergence of the anisotropic sparse grid algorithm: the maximum error given by (15) against the level of sparse grid.
$\sigma_{F}=0.3$. The lognormal random field is obtained through the transformation

$$
F(x)=\exp (\eta+\xi U(x))
$$

with

$$
\begin{aligned}
\eta & =\log \left(\frac{\mu_{F}^{2}}{\sqrt{\mu_{F}^{2}+\sigma_{\mathrm{F}}^{2}}}\right), \\
\xi & =\left(\log \left(1+\frac{\sigma_{\mathrm{F}}^{2}}{\mu_{F}^{2}}\right)\right)^{1 / 2}
\end{aligned}
$$

where $\eta$ and $\xi$ are the location and scale parameters of the lognormal distribution, and $U$ follows a Gaussian distribution with zero mean and the isotropic squared exponential covariance function (24) considering the correlation length $l_{c}=80$.

The Gaussian random field $U$ is discretized through the truncated K-L expansion using 4 terms. The resulting stochastic domain is composed of seven random variables, three of them characterizing the uncertain loads and four of them characterizing the material variability. A non-nested quadrature rule, whose collocation nodes $y^{k}$ are determined by the roots of Hermite polynomials, is used. The nodes and weights of the anisotropic sparse grid are computed adaptively as described above with $N=7, \bar{\ell}=7, \varepsilon=1 \mathrm{e}-2$ and $\ell_{o p t}=3$. The target volume $V^{*}$ is set to $50 \%$ of the initial design domain. The errors for estimating the variance and the mean value of the compliance are $8 \mathrm{e}-3$ and $8 \mathrm{e}-5$, respectively, using 175 sparse grid nodes.

Figure 12 shows the robust designs obtained increasing the value of the parameter $\alpha$. Similarly to previous experiments, these designs show significant topological differences with respect to their deterministic counterpart. As the weighting pa- 


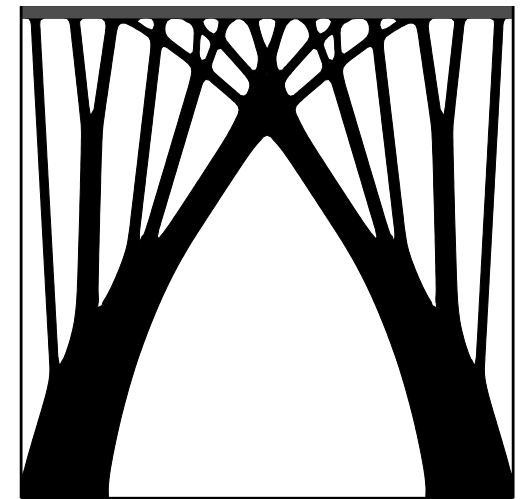

(a) $\alpha=0$

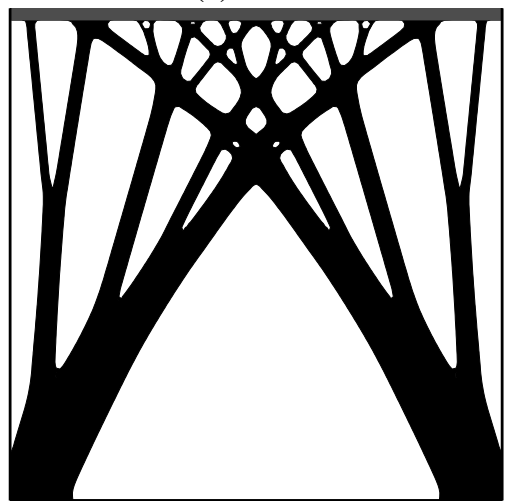

(d) $\alpha=3$

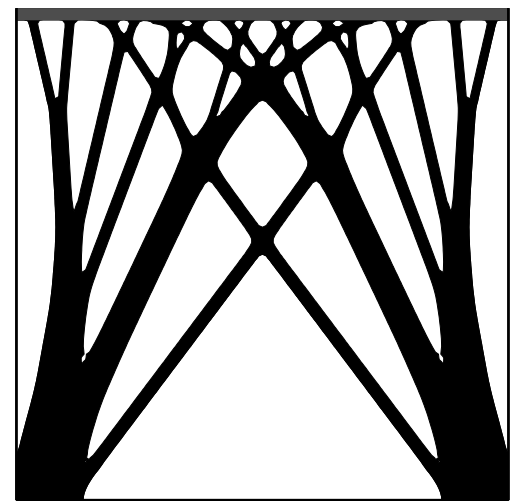

(b) $\alpha=1$

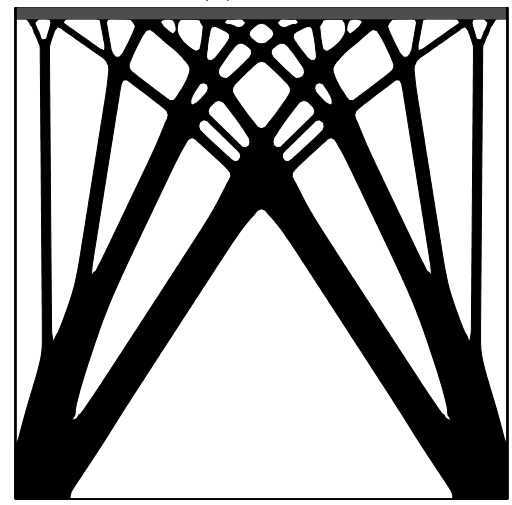

(e) $\alpha=10$

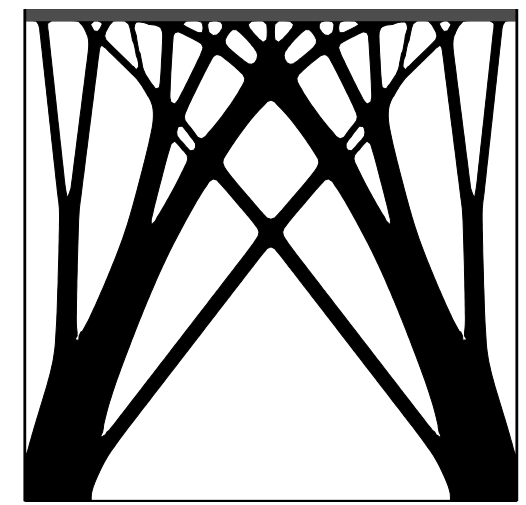

(c) $\alpha=2$

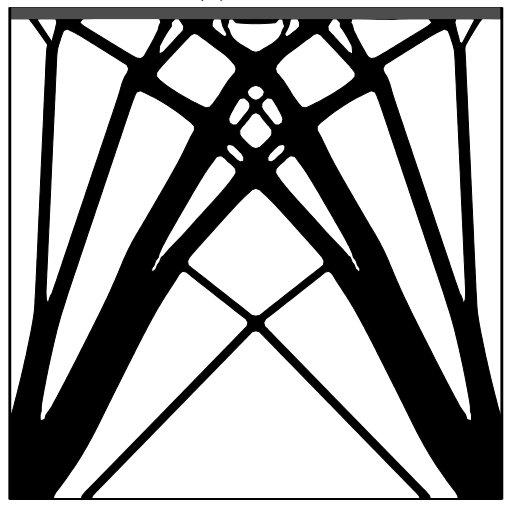

(f) $\alpha=100$

Figure 10: The robust optimized designs of the carrier plate benchmark for different values of $\alpha$.

Table 3: Statistical moments of compliance and volume fraction for different values of $\alpha$ solving the simply supported beam benchmark.

\begin{tabular}{c|ccc}
\hline$\alpha$ & $\mathbb{E}[J]$ & $\operatorname{Std}[J]$ & Vol. frac. \\
\hline Det. & 122.403 & 46.449 & 0.499 \\
0 & 118.970 & 44.748 & 0.502 \\
1 & 120.489 & 43.562 & 0.500 \\
2 & 121.798 & 42.855 & 0.500 \\
3 & 123.113 & 41.968 & 0.501 \\
5 & 126.324 & 41.318 & 0.501 \\
10 & 133.649 & 41.253 & 0.500 \\
\hline
\end{tabular}

rameter $\alpha$ increases, the topologies evolve modifying the number of holes and increasing the thickness of the members. One can also observe that the material distribution is oriented to the left in order to withstand the force with the highest level of uncertainty. Table 3 shows the expected and standard deviation values of the compliance of the optimal designs. We can observe that the optimal designs are the solutions of a multiobjective problem, where a trade-off between the two first statistical moments of compliance is found. This is shown in Figure 6(c) for different values of the $\alpha$ parameter.

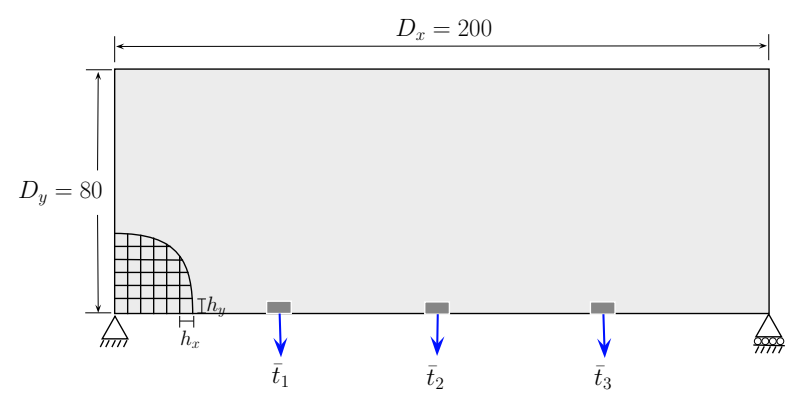

(a)

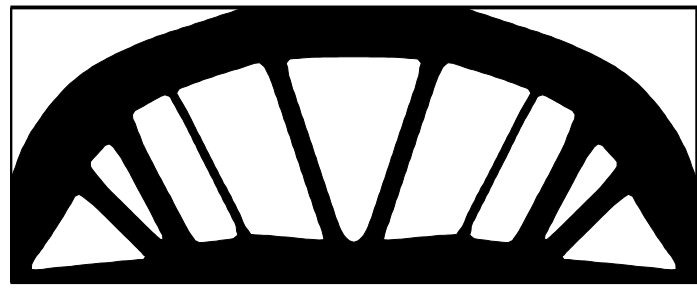

(b)

Figure 11: Simply supported beam benchmark: (a) the design domain and boundary conditions, and (b) the deterministic optimal design. 


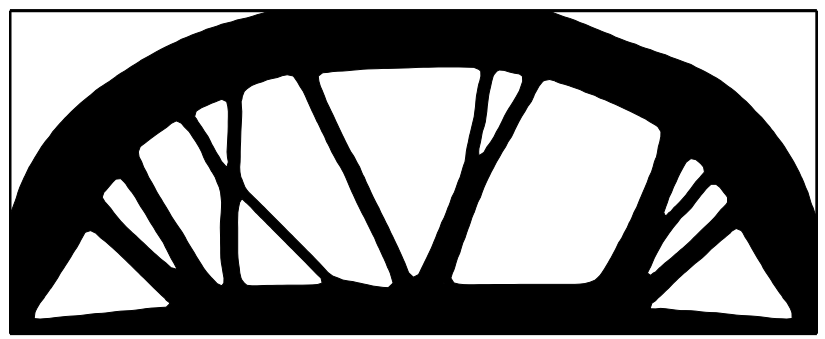

(a) $\alpha=0$

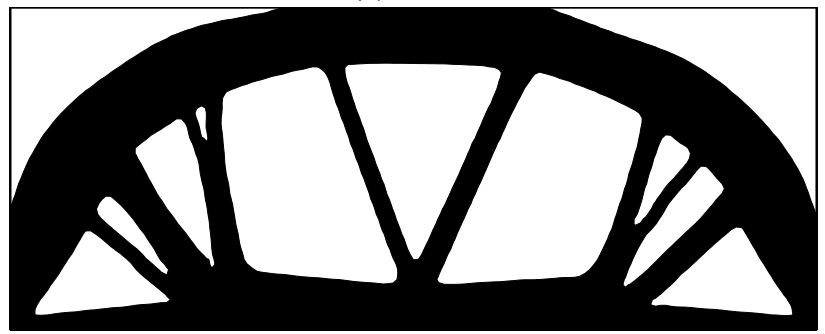

(b) $\alpha=1$

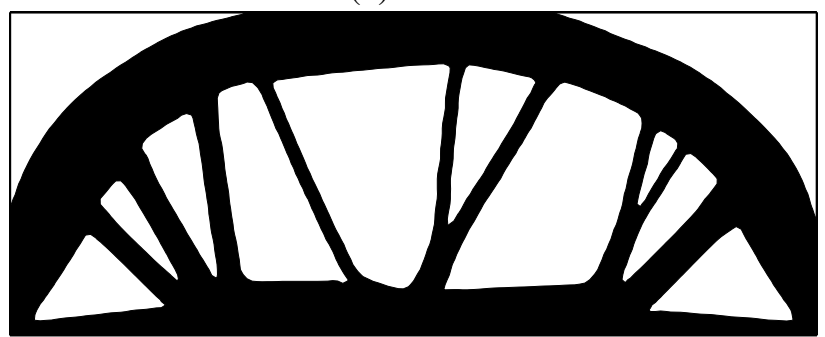

(c) $\alpha=2$

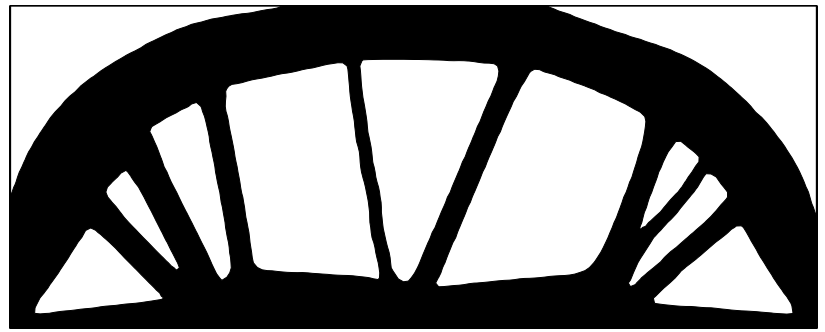

(d) $\alpha=3$

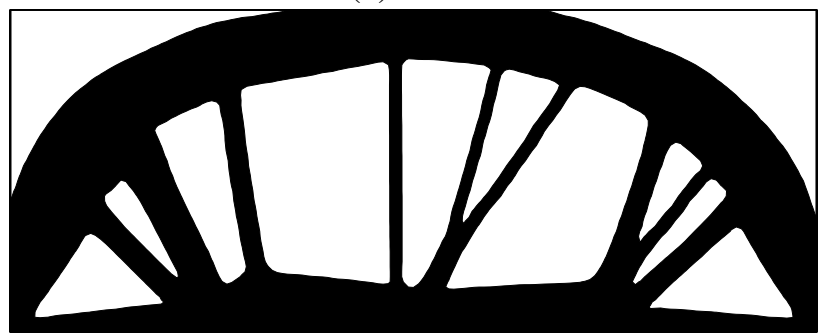

(e) $\alpha=5$

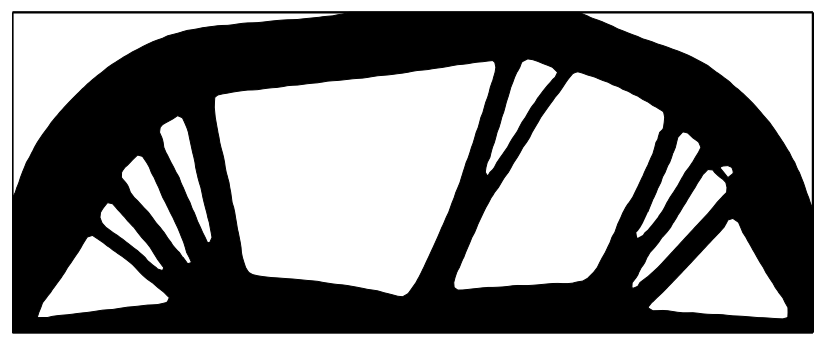

(f) $\alpha=10$

Figure 12: The robust optimized designs of the simply supported beam benchmark for different values of $\alpha$.

\section{Conclusion}

This work proposes a stochastic framework that combines evolutionary methods and sensitivity analysis to address the RTO problem of continuous structures. The proposal addresses the stochastic linear elasticity problem in its weak form, and thus it is not limited by the discretization method or the probabilistic characterization of model uncertainty. The uncertainty of input data can be modeled using random variables with different probability distributions as well as random fields. The method is based on the derivation of an optimality criterion of the RTO problem using a continuous adjoint formulation. The calculation of this optimal criterion involves the estimation of multidimensional integrals over the uncertain parameter space, which is computationally expensive due to the stochastic dimensionality. An adaptive anisotropic sparse grid method is used to solve efficiently the stochastic integrals. The optimality criterion is used as the indicator to identify optimal shapes in an evolutionary optimization algorithm.

The proposal combines the benefits of ETO methods with those of level-set based methods. On the one hand, similarly to level-set methods, a smooth boundary representation is obtained, which facilitates the postprocessing stage. On the other hand, the final topology does not depend on the initial design because new holes are generated during the optimization in regions satisfying the condition $\Phi(\chi, x)<\lambda$. In contrast to other methods addressing the RTO problem, a key contribution of the proposal is that the method provides a trade-off between performance and robustness for different volume fractions during the optimization process. This enables to efficiently find a balance between performance (stiffness of compliance) and robustness (standard deviation) for different volume fractions of material.

The effectiveness of the proposal is evaluated using three RTO benchmarks subjected to loading and material uncertainties. Such experiments include Gaussian and non-Gaussian uncertainties as well as random variables and smooth random fields. We have to remark that the proposal has been particularized to the RTO problem, but it can be extended to other TOUU formulations, such RATO and RBTO.

\section{Acknowledgment}

The authors would like to thank Dr. Francisco Periago for constructive suggestions and discussions. This work has been partially supported by the AEI/FEDER and UE under the contract DPI2016-77538-R and by the "Fundación Séneca-Agencia de Ciencia y Tecnología de la Región de Murcia" under the contract 19274/PI/14.

\section{References}

[1] M. P. Bendsøe, O. Sigmund, Topology Optimization - Theory, Methods, and Applications, second ed., Springer-Verlag Berlin Heidelberg, 2004.

[2] M. P. Bendsøe, N. Kikuchi, Generating optimal topologies in structural design using a homogenization method, Comput. Methods Appl. Mech. Eng. 71 (1988) 197-224.

[3] J. S. Jensen, O. Sigmund, Topology optimization for nanophotonics, Laser Photonics Rev. 5 (2011) 308-21. 
[4] J. H. Zhu, W. H. Zhang, L. Xia, Topology optimization in aircraft and aerospace structures design, Arch. Comput. Meth. Eng. 23 (2016) 595622.

[5] N. Aage, E. Andreassen, B. S. Lazarov, O. Sigmund, Giga-voxel computational morphogenesis for structural design, Nature 550 (2017) 84-6.

[6] O. Sigmund, K. Maute, Topology optimization approaches: A comparative review, Struct. Multidiscip. Optim. 48 (2013) 1031-55.

[7] M. P. Bendsøe, Optimal shape design as a material distribution problem, Struct. Optim. 1 (1989) 193-202.

[8] M. Zhou, G. I. N. Rozvany, The COC algorithm, part II: topological, geometrical and generalized shape optimization, Comput. Methods Appl. Mech. Eng. 89 (1991) 309-36.

[9] G. Allaire, F. Jouve, A.-M. Toader, Structural optimization using sensitivity analysis and a level-set method, J. Comput. Phys. 194 (2004) 363-93.

[10] M.-Y. Wang, X. Wang, D. Guo, A level set method for structural topology optimization, Comput. Methods Appl. Mech. Eng. 192 (2003) 227-46.

[11] M. Burger, R. Stainko, Phase-Field Relaxation of Topology Optimization with Local Stress Constraints, SIAM J. Control Optim. 45 (2006) 144766.

[12] A. Takezawa, S. Nishiwaki, M. Kitamura, Shape and topology optimization based on the phase field method and sensitivity analysis, J. Comput. Phys. 229 (2010) 2697-718.

[13] J. A. Norato, M. P. Bendsøe, R. B. Haber, D. A. Tortorelli, A topological derivative method for topology optimization, Struct. Multidiscip. Optim. 33 (2007) 375-86.

[14] J. Sokolowski, A. Zochowski, On the Topological Derivative in Shape Optimization, SIAM J. Control Optim. 37 (1999) 1251-72.

[15] D. J. Munk, G. A. Vio, G. P. Steven, Topology and shape optimization methods using evolutionary algorithms: a review, Struct. Multidiscip. Optim. 52 (2015) 613-31.

[16] Y. M. Xie, G. P. Steven, A simple evolutionary procedure for structural optimization, Comput. Struct. 49 (1993) 885-96.

[17] O. M. Querin, G. P. Steven, M. Xie, Evolutionary structural optimisation (ESO) using a bidirectional algorithm, Eng. Computations 15 (1998) 1031-48.

[18] M. Victoria, P. Marti, O. Querin, Topology design of two-dimensional continuum structures using isolines, Comput. Struct. 87 (2009) 101-9.

[19] M. Abdi, R. Wildman, I. Ashcroft, Evolutionary topology optimization using the extended finite element method and isolines, Eng. Optimiz. 46 (2014) 628-47.

[20] J. Martínez-Frutos, D. Herrero-Pérez, Gpu acceleration for evolutionary topology optimization of continuum structures using isosurfaces, Comput. Struct. 182 (2017) 119-36.

[21] D. Da, L. Xia, G. Li, X. Huang, Evolutionary topology optimization of continuum structures with smooth boundary representation, Struct. Multidiscip. Optim. (2018) To appear, doi: 10.1007/s00158-017.

[22] R. Ansola, E. Vegueria, J. Canales, J. Tarrago, A simple evolutionary topology optimization procedure for compliant mechanism design, Finite Elem. Anal. Des. 44 (2007) 53-62.

[23] I. Özdemir, Topological derivative based optimization of 3D porous elastic microstructures, Comput. Mater. Sci. 81 (2014) 319-25.

[24] Y. M. Xie, Z. H. Zuo, X. Huang, J. W. Tang, B. Zhao, P. Felicetti, Architecture and urban design through evolutionary structural optimisations algorithms, in: Proc. of the International Symposium on Algorithmic Design for Architecture and Urban Design, 2011.

[25] L. L. Beghini, A. Beghini, N. Katz, W. F. Baker, G. H. Paulino, Connecting architecture and engineering through structural topology optimization, Eng. Struct. 59 (2014) 716-26.

[26] N. D. Lagaros, M. Papadrakakis, Robust seismic design optimization of steel structures, Struct. Multidiscip. Optim. 33 (2007) 457-69.

[27] B. D. Youn, K. K. Choi, R.-J. Yang, L. Gu, Reliability-based design optimization for crashworthiness of vehicle side impact, Struct. Multidiscip. Optim. 26 (2004) 272-83.

[28] M. Schevenels, B. S. Lazarov, O. Sigmund, Robust topology optimization accounting for spatially varying manufacturing errors, Comput. Meth. Appl. Mech. Eng. 200 (2011) 3613-27.

[29] D. Moens, D. Vandepitte, A survey of non-probabilistic uncertainty treatment in finite element analysis, Comput. Meth. Appl. Mech. Eng. 194 (2005) 1527-55.

[30] G. Allaire, C. Dapogny, A linearized approach to worst-case design in parametric and geometric shape optimization, Math. Models Meth. Appl.
Sci. 24 (2014) 2199-257.

[31] F. D. Gournay, G. Allaire, F.Jouve, Shape and topology optimization of the robust compliance via the level set method, ESAIM: Control, Optimisation and Calculus of Variations 14 (2008) 43-70.

[32] Z. Luo, L.-P. Chen, J. Yang, Y.-Q. Zhang, K. Abdel-Malek, Fuzzy tolerance multilevel approach for structural topology optimization, Comput. Struct. 84 (2006) 127-40.

[33] D. W. Kim, B. M. Kwak, Reliability-based shape optimization of twodimensional elastic problems using BEM, Comput. Struct. 60 (1996) 743-50.

[34] S. Conti, H. Held, M. Pach, M. Rumpf, R. Schultz, Risk Averse Shape Optimization, SIAM J. Control Optim. 49 (2011) 927-47.

[35] J. Martínez-Frutos, D. Herrero-Pérez, M. Kessler, F. Periago, Risk-averse structural topology optimization under random fields using stochastic expansion methods, Comput. Methods Appl. Mech. Eng. 330 (2018) 180206

[36] A. T. Beck, W. J. S. Gomes, R. H. Lopez, L. F. F. Miguel, A comparison between robust and risk-based optimization under uncertainty, Struct. Multidiscip. Optim. 52 (2015) 479-92.

[37] J. Zhao, C. Wang, Robust topology optimization under loading uncertainty based on linear elastic theory and orthogonal diagonalization of symmetric matrices, Comput. Methods Appl. Mech. Eng. 273 (2014) 204-18.

[38] M. Tootkaboni, A. Asadpoure, J. Guest, Topology optimization of continuum structures under uncertainty - A polynomial chaos approach, Comput. Methods Appl. Mech. Eng. 201-204 (2012) 263-75.

[39] A. Asadpoure, M. Tootkaboni, J. K. Guest, Robust topology optimization of structures with uncertainties in stiffness - Application to truss structures, Comput. Struct. 89 (2011) 1131-41.

[40] M. Jansen, G. Lombaert, M. Schevenels, Robust topology optimization of structures with imperfect geometry based on geometric nonlinear analysis, Comput. Methods Appl. Mech. Eng. 285 (2015) 452-67.

[41] X. Guo, W. Zhang, L. Zhang, Robust structural topology optimization considering boundary uncertainties, Comput. Methods Appl. Mech. Eng. 253 (2013) 356-68.

[42] J. Martínez-Frutos, D. Herrero-Pérez, M. Kessler, F. Periago, Robust shape optimization of continuous structures via the level set method, Comput. Methods Appl. Mech. Eng. 305 (2016) 271-91.

[43] S. Chen, W. Chen, S. Lee, Level set based robust shape and topology optimization under random field uncertainties, Struct. Multidiscip. Optim. 41 (2010) 507-24

[44] F. Alvarez, M. Carrasco, Minimization of the expected compliance as an alternative approach to multiload truss optimization, Struct. Multidiscip. Optim. 29 (2005) 470-6.

[45] P. D. Dunning, H. A. Kim, G. Mullineux, Introducing Loading Uncertainty in Topology Optimization, AIAA Journal 49 (2011) 760-8.

[46] J. Lógó, B. Balogh, E. Pintér, Topology optimization considering multiple loading, Comput. Struct. (2018) To appear, doi: 10.1016/j.compstruc.2017.03.018.

[47] S.-R. Kim, J. Park, W.-G. Lee, J.-S. Yu, S.-Y. Han, Reliability-based topology optimization based on evolutionary structural optimization, Int. J. Mech. Syst. Sci. Eng. 1 (2007) 135-9.

[48] Y.-S. Eom, K.-S. Yoo, J.-Y. Park, S.-Y. Han, Reliability-based topology optimization using a standard response surface method for threedimensional structures, Struct. Multidiscip. Optim. 43 (2011) 287-95.

[49] K.-H. Cho, J.-Y. Park, S.-P. Ryu, J.-Y. Park, S.-Y. Han, Reliability-based topology optimization based on bidirectional evolutionary structural optimization using multi-objective sensitivity numbers, Int. J. Auto. Tech. 12 (2011) 849-56.

[50] P. Kanakasabai, A. K. Dhingra, An efficient approach for reliability-based topology optimization, Eng. Optimiz. 48 (2016) 1-15.

[51] J. Liu, G. Wen, Y. M. Xie, Layout optimization of continuum structures considering the probabilistic and fuzzy directional uncertainty of applied loads based on the cloud model, Struct. Multidiscip. Optim. 53 (2016) 81-100.

[52] J. Koski, R. Silvennoinen, Norm methods and partial weighting in multicriterion optimization of structures, Int. J. Numer. Meth. Eng. 24 (1987) 1101-21.

[53] K. A. Proos, G. P. Steven, O. M. Querin, Y. M. Xie, Multicriterion evolutionary structural optimization using the weighting and the global criterion methods, AIAA Journal 39 (2001) 2006-12. 
[54] L. Zadeh, Optimality and non-scalar-valued performance criteria, IEEE Trans. Autom. Control. 8 (1963) 59-60.

[55] R. T. Marler, J. S. Arora, The weighted sum method for multi-objective optimization: new insights, Struct. Multidiscip. Optim. 41 (2010) 85362.

[56] G. Hawe, J. Sykulski, Scalarizing cost-effective multi-objective optimization algorithms made possible with kriging, COMPEL 27 (2008) 836-44.

[57] C.-L. Hwang, A. Masud, Multiple Objective Decision Making - Methods and Applications: A State-of-the-Art Survey, volume 164 of Lecture Notes in Economics and Mathematical Systems, Springer-Verlag, Berlin Heidelberg, 1979.

[58] K.-H. Lee, G.-J. Park, Robust optimization considering tolerances of design variables, Comput. Struct. 79 (2001) 77-86.

[59] G. Stefanou, M. Papadrakakis, Assessment of spectral representation and Karhunen-Loève expansion methods for the simulation of Gaussian stochastic fields, Comput. Meth. Appl. Mech. Eng. 196 (2007) 2465-77.

[60] S. A. Smolyak, Quadrature and Interpolation Formulas for Tensor Products of Certain Classes of Functions, Sov. Math. Doklady 4 (1963) 240-3.

61] F. Nobile, R. Tempone, C. G. Webster, A sparse grid stochastic collocation method for partial differential equations with random input data, SIAM J. Numer. Anal. 46 (2008) 2309-45.

[62] F. Nobile, R. Tempone, Analysis and implementation issues for the nu- merical approximation of parabolic equations with random coefficients, Int. J. Numer. Meth. Eng. 80 (2009) 979-1006.

[63] G. Zhang, M. Gunzburger, Error analysis of a stochastic collocation method for parabolic partial differential equations with random input data, SIAM J. Numer. Anal. 50 (2012) 1922-40.

[64] J. Céa, Conception optimale ou identification de formes, calcul rapide de la dérivée directionnelle de la function coût, ESAIM Math. Model. Num. 20 (1986) 371-402.

[65] X. Huang, Y. M. Xie, Convergent and mesh-independent solutions for the bi-directional evolutionary structural optimization method, Finite Elem. Anal. Des. 43 (2007) 1039-49.

[66] F. Jouve, Structural shape and topology optimization, in: G. Rozvany, T. Lewiński (Eds.), Topology Optimization in Structural and Continuum Mechanics, volume 549 of CISM International Centre for Mechanical Sciences, Springer, Heidelberg, 2014, pp. 129-68.

[67] A. Tovar, K. Khandelwal, Uniqueness in linear and nonlinear topology optimization and approximate solutions, in: Proc. of the International Conference on Engineering Optimization, 2010.

[68] A. Aremu, I. Ashcroft, R. Wildman, R. Hague, C. Tuck, D. Brackett, The effects of bidirectional evolutionary structural optimization parameters on an industrial designed component for additive manufacture, Struct. Multidiscip. Optim. 227 (2013) 794-807. 